\title{
Myocardial creatine kinase isoenzyme in serum after subarachnoid haemorrhage
}

\author{
G A V I N F A B I N Y I, D A V I D H U N T, A N D L Y N M C K I L E Y \\ From the Departments of Neurosurgery, Cardiology, and Biochemistry, Royal Melbourne Hospital, \\ Victoria, Australia
}

SUMMARY The myocardial isoenzyme of creatine kinase (MB CK) has been demonstrated in the serum of seven of 16 patients presenting with acute subarachnoid haemorrhage. All patients with elevated MB CK levels exhibited at some stage either intracranial arterial spasm as demonstrated by cerebral angiography, or a focal reversible neurological deficit not due to direct pressure by either intracranial haematoma or aneurysm. The presence of MB CK isoenzymes in sera of patients with subarachnoid haemorrhage may be an index of cerebral ischaemia, and may thus be of value in determining optimal timing of surgery or, ultimately, the long-term prognosis. Changes in the sympathetic nervous system may be a common factor producing both MB isoenzyme release from the myocardium and intracranial arterial spasm.

Many conditions involving the central nervous system, including acute cerebrovascular accident, head injury, meningitis, and encephalitis are known to be associated with elevated serum creatine kinase (CK) levels. (Schiavone and Kaldor, 1965; Dubo et al., 1967; Somer et al., 1975). Doubt remains as to the source of this CK but it is likely to be from skeletal or cardiac muscle rather than from brain tissue. In this report, serum CK was estimated and the isoenzyme detections made in 16 patients with subarachnoid haemorrhage in an attempt to see if cardiac muscle (MB) isoenzyme was present in the peripheral blood, and if its presence correlated with the patient's neurological status.

\section{Patients and methods}

Sixteen patients with subarachnoid haemorrhage admitted to the Royal Melbourne Hospital between October 1974 and December 1975 were studied. In each case the diagnosis was made by the typical clinical findings and confirmed by lumbar puncture. Total cerebral angiography was carried out in 14 patients within the first three days after the bleed. In two patients the angio-

Address for reprint requests: Dr David Hunt, Department of Cardiology, The Royal Melbourne Hospital, Victoria 3050, Australia. Accepted 14 March 1977 graphy was delayed until the sixth day because of the patients' clinical condition. Standard 13 lead electrocardiograms were performed on the first, third, and fifth days.

In each patient total serum CK levels and isoenzyme estimations were determined on the first, third, and fifth days after bleeding. Total CK was estimated on an LKB Wright reaction analyser using a Boehringer packaged kit (Oliver, 1955). The upper limit of normal for this estimation in our laboratory is 80 IU per litre. The presence of isoenzymes was estimated using cellulose acetate electrophoresis after sample application with a millipore applicator. A separation voltage of 200 volts for 15 minutes was applied, and isoenzymes were identified by adding CK reagent (Boehringer) to the strips and reading under ultraviolet light. Qualitative assessment of each isoenzyme present could thus be made (Klein et al., 1973).

Thirty control patients were studied. These patients were in the early postoperative period after abdominal or orthopaedic surgery, and free of known or suspected cardiac disease. The enzyme electrophoresis was performed in both groups of patients by an observer who was unaware of the patients' clinical condition or diagnosis. Creatine kinase levels of over $80 \mathrm{IU} /$ litre were found in 23 patients. MB bands were detected in only three patients, and these had CK 
levels of 940,1325 , and 1744 IU/1 respectively. MB bands were not detected in any of the patients with CK levels under $900 \mathrm{IU} / \mathrm{l}$.

\section{Results}

Raised total CK levels were found in seven of the 16 patients with subarachnoid haemorrhage. MB bands were identified in four of them and also in three of the nine in whom total CK remained within normal limits. There was no correlation between the total CK level and the presence or absence of the MB band.

Electrocardiographic changes occurred in 10 patients; nine patients had ST-T wave changes and another patient had atrial fibrillation. Of these 10 patients, five also had MB bands detected. MB bands were present in two of the six cases without any electrocardiographic abnormalities.

The angiographic studies showed arterial spasm in seven of the 16 patients; MB bands were present in four of these seven, and also in three of the nine patients without spasm. MB bands were identified in all three patients who developed temporary focal neurological signs and in four of 13 patients without such signs.

\section{Discussion}

Three major tissues-brain, skeletal, and cardiac muscle-have been considered responsible for the raised serum CK levels found in some subjects with neurological disorders. This report documents the presence of MB CK isoenzyme in the sera of seven of 16 patients with subarachnoid haemorrhage, and strongly suggests that myocardial damage is responsible for at least some of the $\mathrm{CK}$ in these patients. The presence of $\mathrm{MB}$ isoenzyme in the serum has been shown to be an almost specific indicator of myocardial damage (Wagner et al., 1973; Smith et al., 1976), although raised levels may occur with some patients with myositis (Brownlow and Elevitch, 1974) or muscular dystrophy (Somer et al., 1973), both conditions in which myocardial involvement may occur. Myocardial damage after subarachnoid haemorrhage has been reported in patients (Greenhoot and Reichenbach, 1969: Weidler, 1974) and in experimental animals (Burch et al., 1967; Hunt and Gore, 1972; Weidler, 1974), and the occurrence of electrocardiographic abnormalities has been noted (Abildskov et al., 1970). It has been suggested (Hunt et al., 1969; Cruickshank et al., 1974) that the myocardial damage follows changes in the sympathetic nervous system after intracranial disturbances. MB bands were detected in the serum of three of our control subjects in association with marked elevations of total CK; in none were $\mathrm{MB}$ bands detected with total CK levels below $900 \mathrm{IU} / 1$. Among our subarachnoid haemorrhage patients the highest $\mathrm{CK}$ level recorded was $625 \mathrm{IU} / 1$; it is of interest to note that MB bands were detected in three of the nine patients in whom peak total CK remained within normal limits.

The presence of the BB band, the brain isoenzyme of $\mathrm{CK}$, has been documented in only one of several reports (Dubo et al., 1967; Cao et al., 1969; Somer et al., 1975). Somer and coworkers found BB isoenzymes in low concentrations in the serum of eight of 12 patients with severe acute brain injury, the samples being taken within a few hours of injury. These isoenzymes, however, were not seen in any of seven patients who had major neurosurgical operations. In the same report, significant levels of BB isoenzymes were found in the cerebral venous blood of rabbits after acute severe brain injury induced by the local application of liquid nitrogen; BB isoenzyme levels, however, did not rise in the peripheral venous blood, suggesting either that the total amount of enzyme released was small, or that its clearance was rapid.

All studies have found serum MM (skeletal muscle) isoenzymes to be present when total CK levels are raised in neurological conditions (Dubo et al., 1967; Cao et al., 1969), and there is little doubt that MM CK is the major component of the elevated serum total CK levels. MB bands have not previously been specifically identified which is surprising, but it may be that subarachnoid haemorrhage results in a different pattern of sympathetic output with greater cardiac effects than other neurological conditions.

The occurrence of focal neurological signs such as dysphagia, monoplegia, or cranial nerve dysfunction which subsequently prove to be reversible, has been correlated with local cerebral ischaemia secondary to arterial spasm (Sundt, 1975). In our study five patients had such reversible signs (bilateral abducens palsy, partial oculomotor palsy, dysphasia, trochlear palsy, and hemiparesis). However, in the patient with oculomotor palsy, this was probably due to direct pressure upon the oculomotor nerve from the sac of an aneurysm of the posterior communicating artery. The patient with a dense left hemiparesis had an arteriovenous malformation and associated intracerebral haematoma, and improvement of the neurological signs was delayed until the intracerebral clot was evacuated. Apart from these two patients, it is probable that the reversible deficit 
was largely or wholly consequent upon arterial spasm and local cerebral ischaemia. The three patients with such reversible signs all showed MB bands in their sera.

It is difficult to assess accurately the presence or degree of arterial spasm on a day-to-day basis, as repeated cerebral angiography is impractical and may constitute a hazard to the neurologically ill patient. Until there is a reliable and risk-free method of demonstrating spasm, clinical factors such as level of consciousness or fluctuating neurological signs must be taken into account. The finding of $\mathrm{MB}$ bands in seven of our 11 patients with either angiographic spasm or focal reversible signs as described above, but not in any of the five patients in whom these two factors were absent, suggests an association between spasm and myocardial damage. A common factor may explain the aetiology of both the myocardial damage and the cerebral arterial spasm, and this may be mediated by the sympathetic nervous system. It will require future studies of large numbers of patients to determine if there is any difference in prognosis in subarachnoid haemorrhage patients between those with or without elevated MB CK isoenzyme levels.

\section{References}

Abildskov, J. A., Miller, K., Burgess, M. J., and Vincent, W. (1970). The electrocardiogram and the central nervous system. Progress in Cardiovascular Diseases, 13, 210-216.

Brownlow, K., and Elevitch, F. R. (1974). Serum creatine phosphokinase isoenzyme $\left(\mathrm{CPK}_{2}\right)$ in mycsitis: a report of 6 cases. Journal of the American Medical Association, 230, 1141-1144.

Burch, G. E., Coleclough, M. L., de Pasquale, N. P., and Sohal, R. S. (1967). Acute myccardial lesion following experimentally induced intracranial hemorrhage in mice. Archives of Pathology., 84, 517-521.

Cao, A., de Virgiliis, S., Lippi, C., and Trabalza, N. (1969). Creatine kinase iscenzyme in serum of children with neurological disorders. Clinica Chimica Acta, 23, 475-478.

Cruickshank, J. M., Neil-Dwyer, G., and Stott, A. W. (1974). Possible role of catecholamines, cortico- steroids, and potassium in the production of electrocardiographic abnormalities associated with subarachnoid haemorrhage. British Heart Journal, 36, 697-706.

Dubo, H., Park, D. C., Pennington, R. J. T., Kalbag, R. M., and Walton, J. N. (1967). Serum creatinekinase in cases of stroke, head injury and meningitis. Lancet, 2, 743-748.

Greenhoot, J. H., and Reichenbach, D. D. (1969). Cardiac injury and subarachnoid hemorrhage. Journal of Neurosurgery, 30, 521-531.

Hunt., D., and Gore, I. (1972). Myccardial lesions following experimental intracranial hemorrhage: prevention with propranolol. American Heart Journal, 83, 232-236.

Hunt, D., McRae, C., and Zapf, P. (1969). ECG and serum enzyme changes in subarachnoid hemorrhage. American Heart Journal, 77, 479-488.

Klein, M. S., Shell, W. E., and Sobel, B. E. (1973). Serum creatine phosphokinase isoenzymes after intramuscular injections, surgery and myocardial infarction. Cardiovascular Research, 7, 412-418.

Oliver, I. T. (1955). A spectrophotometric methed for the determination of creatine phosphokinase and myokinase. Biochemical Journal, 61, 116-119.

Schiavone, D. J., and Kaldor, J. (1965). Creatine phcsphokinase levels and cerebral disease. Medical Journal of A ustralia, 2, 790-792.

Smith, A. F., Radford, D., Wong, C. P., and Oliver, M. F. (1976). Creatine kinase MB iscenzyme studies in diagnosis of myocardial infarction. British Heart Journal, 38, 225-232.

Somer, H., Donner, M., Murros, J., and Kenttinen, A. (1973). A serum isoenzyme study in muscular dystrophy. Archives of Neurology (Minneapolis), 29, 343-346.

Somer, H., Kaste, M., Troupp, H., and Konttinen, A. (1975). Brain creatine kinase in blood after acute brain injury. Journal of Neurology, Neurosurgery, and Psychiatry, 38, 572-575.

Sundt, T. M. (1975). Management of ischemic complications after subarachnoid hemorrhage. Journal of Neurosurgery, 43, 418-425.

Wagner, G. S., Roe, C. R., Limbird, L. E., Rosati, R. A., and Wallace A. G. (1973). The importance of identification of myocardial-specific isoenzymes of creatine phosphokinase (MB form) in the diagnosis of acute myocardial infarction. Circulation, 47, 263-269.

Weidler, D. L. (1974). Myocardial damage and cardiac arrhythmias after intracranial hemcrrhage. A critical review. Stroke, 5, 759-764. 